\title{
The Odin orbital observatory
}

H. L. Nordh ${ }^{1}$, F. von Schéele ${ }^{2}$, U. Frisk ${ }^{2}$, K. Ahola ${ }^{3}$, R. S. Booth ${ }^{4}$, P. J. Encrenaz ${ }^{5}$, Å. Hjalmarson ${ }^{4}$, D. Kendall ${ }^{6}$, E. Kyrölä̈ , S. Kwok ${ }^{8}$, A. Lecacheux ${ }^{5}$, G. Leppelmeier ${ }^{7}$, E. J. Llewellyn ${ }^{9}$, K. Mattila ${ }^{10}$, G. Mégie ${ }^{11}$, D. Murtagh ${ }^{12}$, M. Rougeron ${ }^{13}$, and G. Witt ${ }^{14}$

1 Swedish National Space Board, Box 4006, 17104 Solna, Sweden

2 Swedish Space Corporation, PO Box 4207, 17104 Solna, Sweden

${ }^{3}$ National Technology Agency of Finland (TEKES), Kyllikkiporten 2, PB 69, 00101 Helsinki, Finland

${ }^{4}$ Onsala Space Observatory, Chalmers University of Technology, 439 92, Onsala, Sweden

5 Observatoire de Paris, 61 Av. de l'Observatoire, 75014 Paris, France

${ }^{6}$ Canadian Space Agency, PO Box 7275, Ottawa, Ontario K1L 8E3, Canada

7 Finnish Meteorological Institute, PO Box 503, 00101 Helsinki, Finland

8 Department of Physics and Astronomy, University of Calgary, Calgary, ABT 2N 1N4, Canada

9 Department of Physics and Engineering Physics, 116 Science Place, University of Saskatchewan, Saskatoon, SK S7N 5E2, Canada

10 Observatory, PO Box 14, University of Helsinki, 00014 Helsinki, Finland

11 Institut Pierre Simon Laplace, CNRS-Université Paris 6, 4 place Jussieu, 75252 Paris Cedex 05, France

12 Global Environmental Measurements Group, Department of Radio and Space Science, Chalmers, 41296 Göteborg, Sweden

13 Centre National d'Études Spatiales, Centre Spatial de Toulouse, 18 avenue Édouard Belin, 31401 Toulouse Cedex 4, France

${ }^{14}$ Department of Meteorology, Stockholm University, 10691 Stockholm, Sweden

Received 6 December 2002 / Accepted 17 February 2003

\begin{abstract}
Odin is a satellite with a combined astronomy and aeronomy mission. It is designed for observations of species difficult or impossible to observe from ground, especially water and oxygen. The main instrument is a radiometer, operating in the frequency range $486-581 \mathrm{GHz}$ and at $118.75 \mathrm{GHz}$. Its double-reflector telescope has a $1.1 \mathrm{~m}$ primary and the front-end amplifiers are cooled for maximum sensitivity. A 3-axis-stabilisation system provides a pointing accuracy better than $10^{\prime \prime}$. Odin was developed on behalf of the space agencies in Sweden, Canada, France and Finland and was launched into a sun synchronous circular orbit in February 2001. The Odin Science Team, composed of astronomers and aeronomers from the partner countries, has established the observing programme and is responsible for all scientific matters regarding the Odin project. The spacecraft and instruments are performing well and operations are expected to continue well beyond the nominal two-year lifetime.
\end{abstract}

Key words. space vehicles - space vehicles: instruments - telescopes - techniques: spectroscopic

\section{Introduction}

The Odin satellite is operated as an orbital observatory used on equal terms by both astronomers and aeronomers. The astronomical objectives concentrate on the physical and chemical processes in the interstellar medium that relate to star formation. Related and other topics are the composition of comets, atmospheres of giant planets, shells surrounding old stars and spectral line surveys. Key species for the studies are $\mathrm{H}_{2} \mathrm{O}, \mathrm{O}_{2}, \mathrm{NH}_{3}, \mathrm{CI}$ and $\mathrm{CO}$ (Hjalmarson 1997; Nordh 1997; van Dishoeck 1997). Lines of interest occur at submm and $\mathrm{mm}$ wavelengths suffering prohibitive atmospheric absorption.

Send offprint requests to: L. Nordh, e-mail: lennart.nordh@snsb.se
The need for observations above the atmosphere is obvious but it was not until recently possible to build receivers for submm wavelengths suitable for spacecraft.

Odin is equipped with a radiometer sensitive to signals in these almost unexplored wavelength regions (Frisk et al. 2003). The first step in exploring the submm region from space was taken with SWAS (Melnick et al. 1997). Odin is a more sensitive next step, with a larger antenna, and its actively cooled radiometers cover a broader frequency range.

Odin is designed to study line profiles, and thereby velocity fields, in detail and to allow for the use of isotopic lines for accurate abundance determinations. In addition, the wide spectral coverage of the Odin radiometer permits extragalactic observations, molecular searches and unbiased spectral 


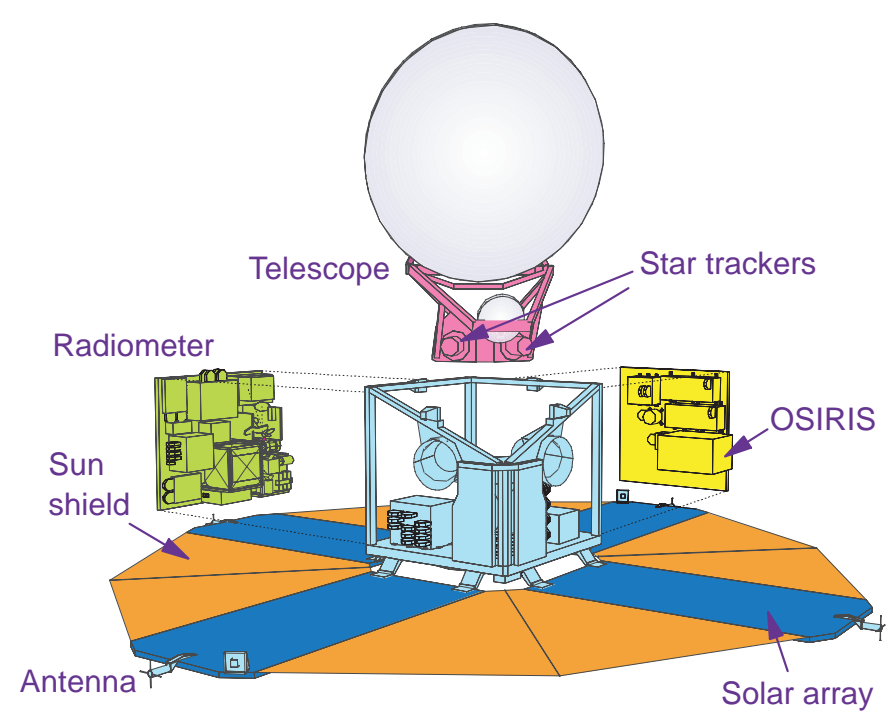

Fig. 1. Odin satellite schematic.

scans (Hjalmarson et al. 2003). Thus, Odin can address many of the scientific issues to be studied by the forthcoming Herschel/HIFI (Pilbratt 2001; de Grauuw et al. 2001).

The aeronomers concentrate on studying the mechanisms behind the ozone layer depletion on a global scale and the mesospheric water vapour distribution. They are interested in some of the same species as the astronomers, with water as the most important common denominator. This made it possible to select radiometer bands that suit both disciplines and fly the joint mission.

The Odin project is a collaboration between Sweden, Canada, Finland and France. Swedish astronomers and aeronomers in 1990 jointly proposed Odin. After scientific and technical preparatory studies and the establishment of a framework of international collaboration, the project was given final go-ahead in 1994. A challenging development period followed whereupon Odin was successfully launched on 20 February 2001, by a START-1 rocket from Svobodny in eastern Russia.

\section{Satellite design}

The basic spacecraft platform functions are to support and to point the scientific instruments at their targets, and to provide power, proper thermal environment, data storage, and communication between on-board equipment and with the ground station. The Odin equipment is accommodated (Fig. 1) on:

- a telescope module, with high thermal stability and alignment accuracy (Sjöberg 1996);

- a bottom deck, where the spacecraft data handling and sensor electronics is placed. The outside houses the launcher interface ring, sun sensors and solar panel mechanisms;

- two vertical decks, one supporting the radiometer equipment, the other supporting the aeronomy instrument OSIRIS and additional spacecraft units.

The satellite mass is $242 \mathrm{~kg}$, the height is $2.0 \mathrm{~m}$, and the width $1.1 \mathrm{~m}$ in stowed configuration and $3.8 \mathrm{~m}$ with deployed solar panels. The main structure is made of aluminium (honeycomb) and the telescope module of carbon fibre reinforced

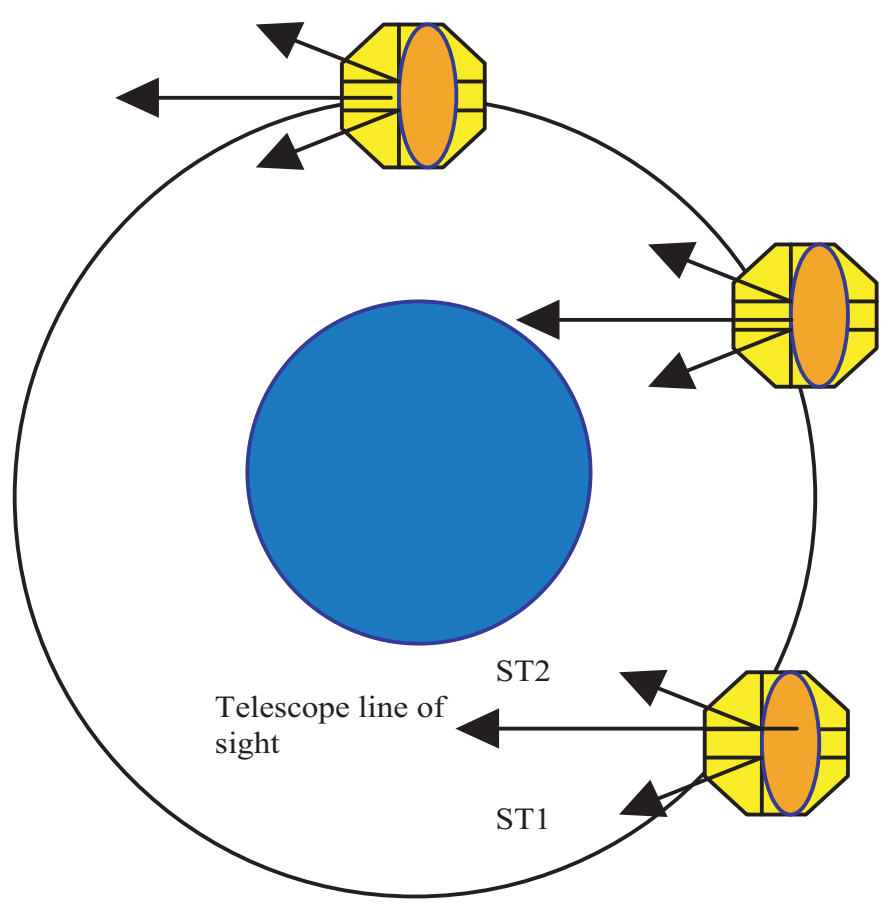

Fig. 2. Astronomy observation geometry. ST1 and ST2 are the linesof-sight of the two star trackers. The polar, sun-synchronous orbit has an altitude of $600 \mathrm{~km}$.

plastics (CFRP). An aluminised kapton sun shield spanned between the solar panels protects the telescope from direct sunlight. Odin generates more than $300 \mathrm{~W}$ in sunlit conditions and relies on batteries for eclipse operations. Measurements are made continuously, except during Earth occultation in astronomy (Fig. 2), and data are stored onboard for later transmission to ground, at a rate of $720 \mathrm{kbps}$. With a total contact time of 80 min per day, up to $300 \mathrm{MB}$ of science data can be downlinked. The on-board System Unit carries out all central spacecraft control, including data handling and power control. It also houses the dedicated attitude control computer. Pointing of the instruments is carried out by moving the entire spacecraft in the two different main modes of observation:

- inertial, long-duration pointing at celestial objects;

- scanning through the earth atmosphere, some 40 times per orbit (limb scanning).

The same, star-referenced, attitude-control system handles both modes (Jakobsson et al. 2002). In addition to the dedicated attitude control computer and its software, the Odin attitude control system includes reaction wheels and magnetic torquers as actuators, and star trackers, gyros, sun sensors and magnetometers as sensors. Full flexibility and redundancy are employed in the platform sub-systems.

\section{Instrument payload}

The Odin scientific payload consists of the submm radiometer (SMR), used by both disciplines, and the optical spectrograph/infrared imaging system (OSIRIS), used by the aeronomers only. 


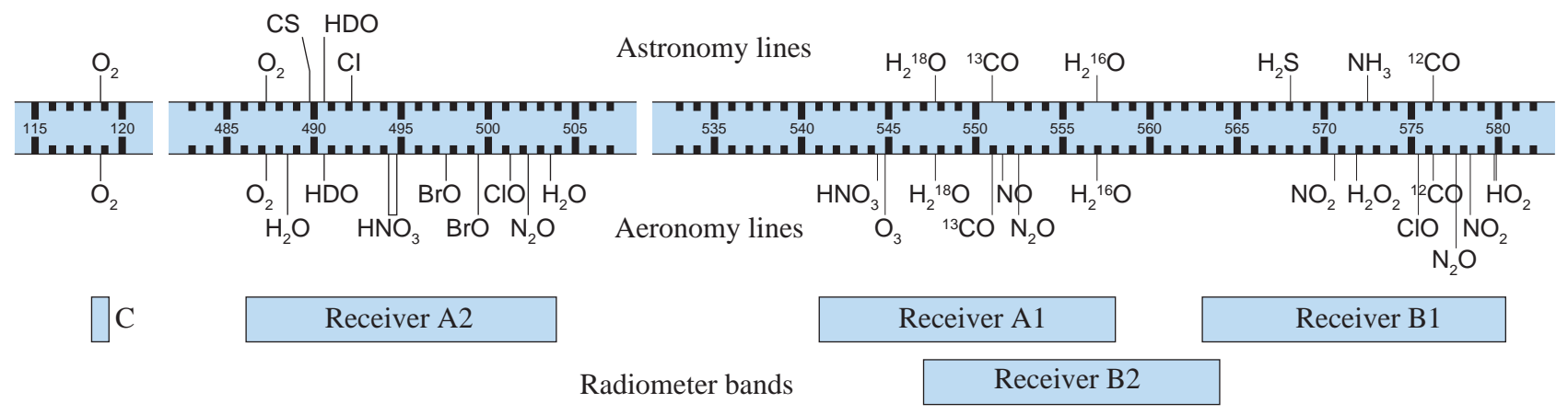

Fig. 3. Radiometer spectral line coverage.

The SMR includes four tuneable submm receivers operating in the frequency range $486-581 \mathrm{GHz}$ and one fixed-tuned $\mathrm{mm}$ wave receiver at $118.75 \mathrm{GHz}$, three spectrometers and a calibration system. The submm receivers cover a bandwidth of $17 \mathrm{GHz}$ each, by means of tuning mechanisms with micron precision and electrically adjustable local oscillators. The frequency resolution is selectable between 0.1 and $1 \mathrm{MHz}$ at instantaneous bandwidths of 0.1 and $1 \mathrm{GHz}$, respectively. All receivers have the same viewing direction and are fed by a CFRP offset Gregorian telescope. The measured rms surface accuracy of the $1.1 \mathrm{~m}$ diameter main reflector and the subreflector is $8 \mu \mathrm{m}$ and $5 \mu \mathrm{m}$, respectively. The errors in the alignment, together with the effects of the in-orbit environment, result in an estimated loss of efficiency of $0.1 \mathrm{~dB}$. The beam-size is on the order of $2^{\prime}$ for the high frequency receivers and $10^{\prime}$ for the $119 \mathrm{GHz}$ receiver. The telescope is designed to combine low side-lobes, high gain and thermal stability. All receivers (mixers and pre-amplifiers) are cooled for maximum sensitivity. The anticipated (and now confirmed) low abundance of $\mathrm{O}_{2}$, led to the inclusion of a dedicated $119 \mathrm{GHz}$ receiver equipped with a sensitive 3 -stage transistor (HEMT) preamplifier. Two small, low-power, hybrid autocorrelation spectrometers and one acousto-optic spectrometer are used as back-ends. The radiometer can be operated in a variety of modes. Normally two submm receivers and the $119 \mathrm{GHz}$ receiver are operated in parallel (Frisk et al. 2003).

In Fig. 3 the positions of the most important spectral lines, covered by the radiometer, are displayed. The spectral coverage is a compromise between astronomy and aeronomy needs and led to the exclusion of the stronger $\mathrm{O}_{2}$ line at $425 \mathrm{GHz}$. Spectra are normally read out every $10 \mathrm{~s}$ but the stability of the radiometer allows very long total integration times to be used; up till now the longest integration time is 100 hours, corresponding to approximately the same number of orbits. Reference switching against cold sky is made through beam switch optics or through the telescope by redirecting the spacecraft.

The radiometer was designed for flexibility in order to be adaptable to changing scientific requirements and to overcome possible failure or degradation of parts of the receivers.

\section{Orbit and operations}

The Odin orbit is sun synchronous (near polar) and circular at $600 \mathrm{~km}$ altitude, with the ascending node at 18:00 hours. The period is $97 \mathrm{~min}$. This orbit fulfils the requirements of both scientific disciplines, e.g. full sky coverage twice a year for astronomy. It is stable for several more years than the specified two-year lifetime of Odin. Measurements are made throughout the orbit in aeronomy but in astronomy the Earth occults the telescope line-of-sight during 30 min per orbit. This time is used for calibrations, new settings of the radiometer etc. Data are stored in a solid state memory for later transmission to the Swedish Space Corporation (SSC) Esrange ground station in northern Sweden. Odin is visible from the station about 80 min per day spread over $10-11$ passes. The satellite is preprogrammed to make its observations, up to several days in sequence, without the need for operator intervention (Lundin 2002).

The observing programme is defined by the scientific community using guidelines and $\mathrm{S} / \mathrm{W}$ tools regarding the physical constraints of the satellite. The astronomy programme constraints, e.g. source visibility and solar aspect angles, are handled by a planning tool developed in Canada and Sweden to suit the Odin mission. For practical operations the observing programme is broken down into timelines of about four days each. The timelines are validated by the Mission Control Centre (MCC), a long-term planning and analyses group consisting of the core satellite development team, at SSC Solna, Sweden. Head of the group is the Mission Manager who is responsible for the operations of Odin and the operational interface to the scientific community. The MCC also takes care of performance and anomaly analyses. The Odin Control Centre (OCC) at SSC Esrange handles all daily operations tasks. The OCC performs the last validation of the timeline based on fresh orbital data, translates the final timeline into commands and transmits it to the satellite. After reception of scientific and housekeeping data from the satellite the OCC performs post-processing to reconstruct the orbit and the attitude of the spacecraft. All science data, including time tags and pointing information, are archived on a central server and made available to the instrument data centres through the Internet. Data processed by the instrument centres and the science groups are archived on the same central server, and made available at various levels of processing.

\section{Project organisation and development}

The Odin project is managed by SSC, acting on behalf of the Swedish National Space Board (SNSB) supported by its international partners, the Canadian Space Agency (CSA), 
the National Technology Agency of Finland (Tekes) and the Centre National d'Études Spatiales (CNES), France. In the beginning of the project agreements were made on the economic and programmatic boundary conditions and the technological content of the contribution from each country, in three bilateral Memoranda of Understanding. The Odin Science Team, common for both astronomy and aeronomy, set the scientific requirements, advised the SSC Project Team throughout the development phase and is responsible for all scientific matters during the operations phase. The Science Team consists of the SNSB Programme Scientist (Chair), the SSC Project Scientist/Mission Manager, the Astronomy and Aeronomy Mission Scientists and eight additional astronomers and aeronomers representing the participating countries. Members of the Science Team act as leaders of national teams of appointed Odin scientists, associated scientists and instrument scientists.

SSC was the prime contractor for development and launch of Odin and is also responsible for the operations of the satellite, under direct contract from SNSB. The SMR Data Centre at Chalmers/Onsala in Sweden is responsible for the first level of processing and validation of the scientific data. The science groups further refine the data in order to extract useful information.

As prime contractor, SSC was responsible for satellite system and subsystem design, development, procurement, integration and testing. In addition, SSC contributed to the design of the SMR and managed its development and testing. Development was carried out in a low-cost approach (v. Schéele 2002), using a small project team, few management layers, and with an emphasis on testing over analysis.

\section{Observing time and programme}

The available observing time is shared equally between astronomy and aeronomy, although the timeline requirements of the two disciplines are quite different. The aeronomers need global coverage at regular and frequent intervals. At certain times of the year they require continuous observations of the atmosphere for up to two weeks, but it is not possible to predict accurately when these periods will occur. The astronomy programme has source visibility, source crowding, sky-beam positions and the solar aspect angle as the main constraints.

The species that can be observed with Odin occur in a wide variety of astronomical targets and regions and therefore the observing programme has been planned around a number of specific topics. These topics, based on separate proposals to the Odin Science Team, have been harmonised in order to create a coherent programme with a minimum of duplication and no gaps. Each topic is studied by a team of Odin astronomers from all the participating countries, guided by two Lead Scientists. The topical team is responsible for the preparatory work, the evaluation of the early results and the data analysis regarding its topic. The results of all the topics are available to, and also limited to, the entire team of Odin astronomers. An observing programme covering many months in advance is formally decided by the Odin Science Team. When changes to the decided and on-going programme are called for, action is taken by a small executive group consisting of the Programme Scientist, the Mission Manager, the Aeronomy Mission Scientist and the Astronomy Mission Scientist. In case of major revisions the group alerts the Odin Science Team.

Odin is well suited for galactic observations and thus most of the astronomy observing time so far has been used for studies of the galactic interstellar medium with a focus on regions of star formation. Efforts to detect $\mathrm{O}_{2}$ have been the main driver of very long integration times. A large fraction of the time has also been spent on detailed studies of four comets. Less time has been spent on evolved stars with circumstellar shells. NGC 253 and LMC are some extragalactic objects that have been observed. Dedicated spectral scans were not yet scheduled, but the long integration times dedicated to $\mathrm{O}_{2}$ have resulted in sensitive searches within limited frequency regions around the main target lines. A simultaneous search in Orion for ${ }^{15} \mathrm{NH}_{3}$ and $\mathrm{PH}$ has been performed.

\section{In-orbit status}

The launch of Odin, on February 20, 2001 at 17:48 local time on a START-1 launcher was nominal and the injection at 18:04 into the sun synchronous, $600-\mathrm{km}$ altitude orbit was well within the specification. The instruments were delivering test data within the first week. Jupiter measurements were made in March, comet Linear-A was studied in April and first measurements in aeronomy mode were made in May. Commissioning of the satellite, notably the attitude control system, went on continuously in parallel with generation of science data. By August uninterrupted high-accuracy pointing had been established for astronomy. The in-flight pointing accuracy $\left(10^{\prime \prime}\right.$ in astronomy) and pointing stability (4" during one hour) is well within the requirements. The pointing efficiency, i.e. stable pointing with sufficient certainty in the reconstruction, during scheduled time is better than $95 \%$.

Problems to start the mechanical cooler in the beginning of the mission could be overcome to allow for cooling of the receivers. Alignment and frequency stability of the receivers are mostly excellent. The system noise temperature of the receivers (submm receivers $3300 \mathrm{~K}$, and the $\mathrm{mm}$ receiver $600 \mathrm{~K}$ ) is as expected from the pre-launch laboratory tests. Phase lock problems in two of the channels, $119 \mathrm{GHz}$ and $572 \mathrm{GHz}$, are compensated for in the data processing by using atmospheric lines during the occulted part of the orbit to determine the frequency drift. Using Jupiter the in-orbit telescope properties and the pointing capability were determined and found to be excellent and close to theoretical expectations. Odin has targeted a variety of astronomical objects, including several comets. Among other things Odin has:

- detected the ground transition of $\mathrm{NH}_{3}$ in molecular clouds; - for the first time measured the isotopic ratio of $\mathrm{H}_{2}{ }^{16} \mathrm{O}$ to $\mathrm{H}_{2}{ }^{18} \mathrm{O}$ in a comet;

- lowered the detection limits of $\mathrm{O}_{2}$;

- mapped water vapour outflow in e.g. Orion.

All in all, the observatory fulfils the demanding technical requirements set out at the start of the project. Both scientific and operational (e.g. attitude reconstruction) data processing 
on ground has been increasingly automated since the launch. Odin is presently turning out up to $300 \mathrm{MB}$ of data per day to astronomers and atmospheric researchers in the four countries. There is reason to believe that this can continue well beyond the two-year design-goal lifetime since the orbit is stable within expectations, the instruments are working well, and no redundancy switch-in of spacecraft equipment has yet been necessary.

\section{Conclusions}

The Odin project demonstrates that a small, low-cost scientific mission can successfully address important scientific questions and prepare for tomorrow's challenges. The excellent in-flight performance, present spacecraft and payload status and the quality of the results obtained so far justify the recent decision by the four partner country agencies to continue the observations, until April 2004 nominally. A continued observing programme based on the findings of the first 20 months in orbit holds promise for significant scientific progress in the coming year(s).

Acknowledgements. Since the start of the Odin activities in 1990, a large number of engineers and scientists have contributed to its development, launch and operations. Without their expertise, enthusiasm, dedication, professionalism and hard work, the success of Odin would not have been possible. We particularly want to mention G. Olofsson, who was one of the initiators of the project and has remained a key person since then. On the astronomy side we also want to acknowledge the contributions to the project by M. Olberg, Aa. Sandqvist, S. Torchinsky and K. Volk, who have been of vital importance for the success of the mission. We also want to thank the space agencies of the participating countries and their respective advisory space science committees for a good collaboration and a never failing support during the entire development time. Special thanks go to K. Fredga, for resolutely guiding Odin from proposal to decision, and to the SSC project team for dedicatedly realising the project. Finally the Knut and Alice Wallenberg Foundation is thanked for a generous contribution to the development of the Odin radiometer.

\section{References}

van Dishoeck, E. F. 1997, in Proc. of the ESA Symp., The far-infrared and submillimetre Universe, ESA SP-401, 81

Frisk, U., Hagström, M., Ala-Laurinaho, J., et al. 2003, A\&A, 402, L27

de Grauuw, T., \& Helmich, F. P. 2001, in Proc. ESA Symp., The Promise of the Herschel Space Observatory, ESA SP-460, 45

Hjalmarson, Å. 1997, in Proc. IAU Symp. 170 (1995), CO: Twentyfive years of millimetre wave spectroscopy, ed. W. B. Latter et al. (Kluwer Academic Publishers), 227

Hjalmarson, Å., Frisk, U., Olberg, M., et al. 2003, A\&A, 402, L39

Jakobsson, B., Nylund, M., Olsson, T., et al. 2002, 53rd International Astronautical Congress, The high-performing attitude control on the scientific satellite Odin, IAC-02-A., P. 13

Lundin, S. 2002, 53rd International Astronautical Congress, Finding the balance between autonomy on-board versus man-triggered actions from ground, IAC-02-IAA[1].11.4.04

Melnick, G. J., Dalgarno, A., Erickson, N. P., et al. 1997, in Proc. ESA Symp., The far-infrared and submillimetre Universe, ESA SP-401, 189

Nordh, H. L. 1997, in Proc. ESA Symp., The far-infrared and submillimetre Universe, ESA SP-401, 195

Pilbratt, G. 2001, in Proc. ESA Symp., The Promise of the Herschel Space Observatory, ESA SP-460, 13

v. Schéele, F. 2002, 53rd International Astronautical Congress, The Odin project: Lessons for a Follow-on EO Mission, IAC-02IAA.11.2.03

Sjöberg, F. 1996, 47th International Astronautical Congress, The design and manufacturing of a thermally stable submillimetre wave space-borne telescope, IAF-96-I.1.11 